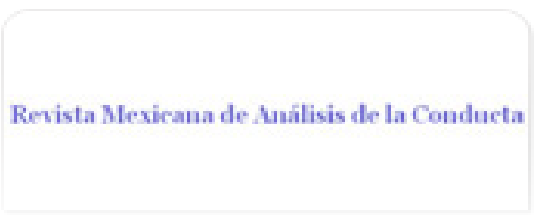

Revista Mexicana de Análisis de la Conducta
Revista Mexicana de Análisis de la Conducta

ISSN: 0185-4534

editora@rmac-mx.org

Sociedad Mexicana de Análisis de la Conducta

México

SILVA VICTORIA, HÉCTOR OCTAVIO; MORALES CHÁVEZ, GERMÁN; PACHECO CHÁVEZ, VIRGINIA; CAMACHO TREJO, AURA GABRIELA; GARDUÑO ORTUÑO, HÉCTOR MIGUEL; CARPIO RAMÍREZ, CLAUDIO ANTONIO DIDÁCTICA COMO CONDUCTA: UNA PROPUESTA PARA LA DESCRIPCIÓN DE LAS HABILIDADES DE ENSEÑANZA

Revista Mexicana de Análisis de la Conducta, vol. 40, núm. 3, diciembre, 2014, pp. 32-46 Sociedad Mexicana de Análisis de la Conducta Distrito Federal, México 


\title{
DIDÁCTICA COMO CONDUCTA: UNA PROPUESTA PARA LA DESCRIPCIÓN DE LAS HABILIDADES DE ENSEÑANZA
}

\author{
DIDACTIC AS BEHAVIOR: A PROPOSAL FOR THE DESCRIPTION \\ OF TEACHING SKILLS
}

\section{HÉCTOR OCTAVIO SILVA VICTORIA, GERMÁN MORALES CHÁVEZ, VIRGINIA PACHECO CHÁVEZ, AURA GABRIELA CAMACHO TREJO, HÉCTOR MIGUEL GARDUÑO ORTUÑO Y CLAUDIO ANTONIO CARPIO RAMÍREZ \\ UNIVERSIDAD NACIONAL AUTÓNOMA DE MÉXICO, FACULTAD DE ESTUDIOS SUPERIORES IZTACALA}

\begin{abstract}
Resumen
En el presente trabajo se describe el Modelo Interconductual de Desempeño Didáctico (MIDD) en concordancia con una descripción de desarrollo psicológico. El MIDD propone que el ejercicio que tiene quien enseña en la interacción didáctica puede ser ubicado en siete ámbitos de desempeño en los cuales se desarrollan habilidades y competencias específicas: 1) La planeación didáctica, 2) la exploración competencial, 3) la Explicitación de criterios, 4) la ilustración, 5) la supervisión de la práctica, 6) la retroalimentación, y 7) la evaluación. Cada uno de estos ámbitos es descrito en términos de habilidades conductuales: de acuerdo al tipo de desempeño que implican, el propósito que se persigue tal desempeño y la situación en que ese desempeño es funcional. Finalmente, se describen los resultados generales de algunos estudios derivados del MIDD así como sus implicaciones para investigaciones futuras y potenciales aplicaciones.

Universidad Nacional Autónoma de México, Facultad de Estudios Superiores Iztacala. Av. De los Barrios No. 1, Los Reyes Iztacala. Tlalnepantla, Estado de México. C.P. 54090. Correos electrónicos: Germán Morales: gmoralesc@unam.mx; Virginia Pacheco: vpacheco@unam.mx; Aura Camacho: aura.camacho@hotmail. com; Héctor Garduño: hector.garduno@hotmail.com; Claudio Carpio: carpio@unam.mx

Todos los autores colaboraron de manera equitativa en la realización del presente escrito.

Este trabajo fue posible gracias al financiamiento proporcionado por la DGAPA a través de los programas PAPIME 301512, PAPCA 2011-2012 No. 36 y PAPIIT IN304810.
\end{abstract}


Palabras clave: interacción didáctica, desarrollo psicológico, habilidades de enseñanza, ámbitos de desempeño didáctico.

\begin{abstract}
This paper proposes the Didactic Performance Interbehavioral Model (DPIM) in agreement with a description of psychological development. The DPIM proposes that didactic interaction of one who teaches may be located in seven areas of performance in which skills and competencies are developed: 1) Didactic planning, 2) competence exploration, 3) explicitation of criteria, 4) illustration, 5) practice supervision, 6) feedback, and 7) evaluation. Each of these areas is described in terms of behavioral skills: according to the type of performance involved, the purpose intended with this performance and the situation in which such performance is functional. The general results derived from DPIM studies and their implications for future research and potential applications are described.

Keywords: didactic interaction, psychological development, teaching skills, areas
\end{abstract} of didactic performance

Un momento importante en la evolución de los homínidos fue cuando comenzaron a usar elementos de la naturaleza como herramientas para sobrevivir. Según Engels (1988) las herramientas se constituyeron como una extensión o prolongación especializada de la mano que permitió una mayor eficacia en el trabajo y su diversificación. El uso de las herramientas generó un aumento en la complejidad de las prácticas, por lo que fue necesario que los homínidos modularan la conducta de otros con base en su propia experiencia. De esta forma incrementó la probabilidad de sobrevivir como especie y se fortaleció la esencia de los homínidos como seres sociales. Dada esta característica social, la preservación del ser humano no se reduce a la mera reproducción biológica de la especie, sino que consiste en la reproducción y perfeccionamiento de las prácticas colectivas e individuales, en las que está implicado el uso de las herramientas como conducta y sus productos (López, 1994; Ribes, 1990).

En otras palabras, los seres humanos no sólo han usado herramientas en sus prácticas sino que se han vuelto cada vez más hábiles en conseguir que otros las usen al incorporarse a la sociedad. Esta división es fundamental para comprender la relevancia de la educación como un mecanismo de transformación social. Dos individuos son esenciales en la descripción de este fenómeno educativo: a) aquel individuo que será incorporado a las prácticas sociales, quien aún no es capaz de realizarlas, y b) aquel que por el dominio que tiene sobre alguna práctica social está en condiciones de incorporar a otros mediante alguna estrategia. Cuando este fenómeno ocurre en instituciones formalizadas como la escuela en- 
tonces los individuos mencionados son identificados como el alumno y el profesor.

Es posible realizar diversos tipos de descripción sobre: a) las características de quien aprende, b) las características de quien enseña, y c) las características de la relación que mantienen estos individuos. Adicionalmente, se pueden realizar estas descripciones con base en diferentes dimensiones de análisis. Por ejemplo, se puede realizar un análisis histórico social acerca de la manera en que ha ido evolucionando la relación entre ellos. Es posible realizar un análisis económico acerca del costo que implica la proporción entre la cantidad de profesores y alumnos en las instituciones educativas de una sociedad en particular. También es posible realizar un análisis político relacionado con el tipo de normas y reglas a las que debe apegarse la relación entre estos individuos. También es posible una reflexión epistemológica, acerca de cómo se relacionan ambos individuos con eso que llamamos conocimiento. Por supuesto, entre los tipos de análisis posibles se encuentra el psicológico o conductual, que recupera la evolución ontogenética de cada individuo en esta relación. Conviene ser consciente de las fronteras analíticas existentes para no cometer la imprudencia de fusionar términos de distintas dimensiones analíticas en lo que Ryle (2005) denominó errores categoriales.

El propósito de este manuscrito es presentar una propuesta para describir en una dimensión psicológica a uno de estos individuos, aquel que por el dominio que tiene de alguna práctica está en condiciones de incorporar a otro que no puede realizarla. Específicamente, se propondrá una manera de describir la conducta de enseñar o conducta didáctica en términos de las diferentes habilidades que se ponen en juego para conseguir que otro individuo aprenda algo. El trabajo es una descripción del modelo y de los estudios que se han realizado para probarlo. Al concentrar el análisis en la conducta del individuo que enseña no se está negando la existencia de quien aprende, ni de aquello que hay que enseñar, ni de las circunstancias en las que se enseña y se aprende. Por el contrario, el análisis del desarrollo de las habilidades didácticas implica incorporar estos elementos a la explicación psicológica. Es conveniente hacer esta precisión para concentrarse en quien enseña considerando su comportamiento en un marco más amplio de análisis de interacción didáctica.

La propuesta analítica que se presenta se fue gestando poco a poco desde 1998, año en que el Dr. Carpio y sus colaboradores realizaron un análisis para describir la relación entre los paradigmas y las prácticas científicas, tecnológicas y pedagógicas (Carpio, Canales, Pacheco, \& Flores, 1998). Ese trabajo sirvió como plataforma para que entre el año 2003 y 2004 el Dr. Carpio y el Dr. Ibáñez coordinaran la actualización curricular en varias de las carreras de la Universidad de Sonora. Se fortaleció entonces el interés por reconocer la dimensión conductual de la práctica docente que, como será descrito más adelante, se fue realizando en concordancia con una descrip- 
ción sobre el desarrollo conductual de los individuos. (Carpio, Pacheco, Canales, \& Flores, 2005).

\section{Sobre el desarrollo conductual de los individuos.}

Desde la perspectiva Interconductual, el desarrollo psicológico de los individuos se describe como la evolución conductual en tres tipos de situaciones, que difieren entre sí por la especificidad, ambigüedad o inexistencia de criterios conductuales por

\section{Situaciones Contingencialmente Cerradas (SCC)}

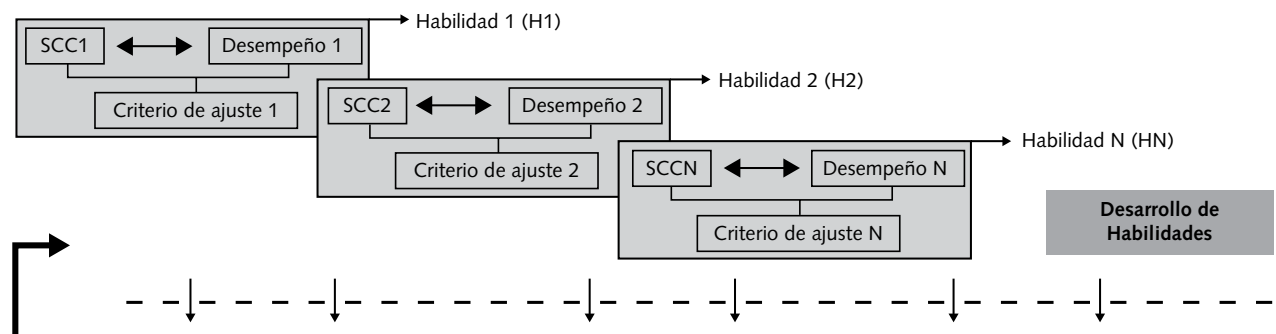

Situaciones Contingencialmente Abiertas (SCA)
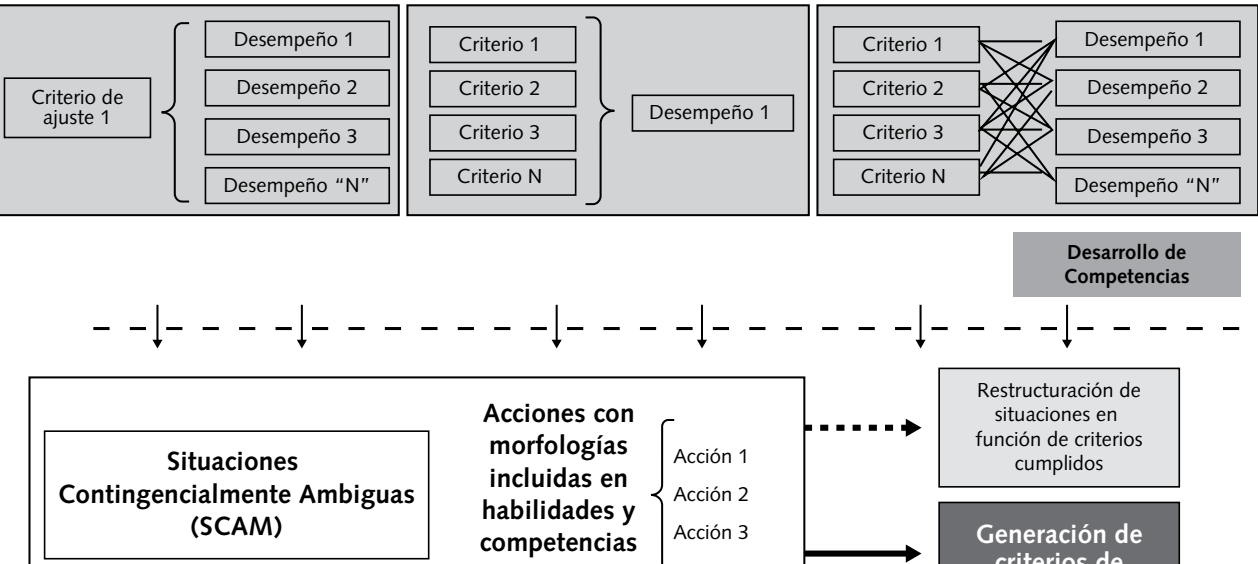

$\begin{aligned} & \text { Acciones con } \\ & \text { morfologías } \\ & \text { incluidas en } \\ & \text { habilidades y } \\ & \text { competencias } \\ & \text { previas }\end{aligned}$
Acción 2
Acción 3

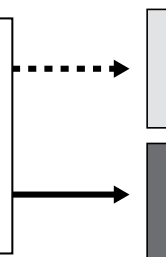

Restructuración de situaciones en función de criterios cumplidos

\section{Generación de}

criterios de ajuste

Comportamiento Creativo

Figura 1. Modelo Interconductual del Comportamiento Creativo (tomado de Carpio, Canales, Morales, Arroyo, \& Silva, 2007). 
satisfacer en ellas. En la Figura 1 se presenta el Modelo Interconductual del Comportamiento Creativo (MICC), que propusieron Carpio, Canales, Morales, Arroyo y Silva (2007), que se constituye como la descripción interconductual del desarrollo psicológico. El MICC describe que los individuos inician su desarrollo psicológico en situaciones contingencialmente cerradas. En la primera de las situaciones existe un criterio conductual por satisfacer y sólo hay una forma de satisfacerlo. La correspondencia precisa entre criterio-situación-desempeño es lo que comúnmente se denomina habilidad. Una habilidad es la integración funcional de estos tres elementos y, en las situaciones contingencialmente cerradas, los individuos tienden a desarrollarlas. Alguien es hábil cuando puede hacer o decir lo que se requiere en una situación precisa para cumplir un criterio específico. Este modelo insiste en reconocer que habilidad no se reduce a actividad, porque lo que los individuos hacen o dicen sólo tiene sentido psicológico en función del criterio o demanda por satisfacer.

En la medida en la que el individuo desarrolla habilidades su repertorio conductual se diversifica y surge la posibilidad de interactuar en situaciones contingencialmente abiertas en las que: a) se puede satisfacer un criterio conductual de varias formas posibles, b) se pueden satisfacer varios criterios conductuales con base en el mismo tipo desempeño, y c) se pueden satisfacer múltiples criterios con diversas formas de desempeño. Cuando un individuo puede variar su desempeño sin depender de una forma particular para el cumplimiento de criterios conductuales se dice que es competente. Esta clase de conducta es lo que comúnmente se denomina inteligencia.

Finalmente, en la medida en la que se desarrollan las competencias conductuales existe la posibilidad de interactuar en situaciones contingencialmente ambiguas. En estas situaciones no existe un criterio conductual a satisfacer, pero puede haberlo si el individuo con su actividad lo genera. Es decir, la conducta en este tipo de situaciones ya no consiste en el cumplimiento de los criterios, ni de manera estereotipada ni de manera variada, sino en dar origen a criterios. Esta conducta es lo que comúnmente llaman creatividad. Surge así la posibilidad de que el mismo individuo u otros desarrollen a partir de los criterios generados habilidades y competencias que no se habían observado antes. Como puede apreciarse en esta descripción no parece concebible la conducta creativa sin el desarrollo de la conducta inteligente, pero tampoco es posible ser inteligente sin el desarrollo de habilidades.

Existen algunos comentarios adicionales que realizar acerca del MICC. Primero, en el análisis de la conducta de un individuo la secuencia de su desarrollo en diferentes tipos de situaciones debe ser ubicada en un ámbito específico de desempeño de la actividad humana. Segundo, la vida de los individuos debe describirse como el desarrollo conductual en múltiples ámbitos de desempeño y no sólo en uno. Tercero, los criterios conductuales por satisfacer son específicos de cada ámbito de desempeño de actividad humana, por lo tanto provienen de la sociedad y su historia, por lo 
que son distintos entre épocas y entre regiones. Cuarto, el desarrollo psicológico no es simétrico ni tiene la misma celeridad entre los distintos ámbitos, lo que permite entender que como individuos seamos hábiles en un ámbito, inteligentes en otros, creativos en otros más, etc. Resulta absurdo desde esta perspectiva suponer que alguien es creativo en todo, o inteligente en todo, o hábil en todo.

En este análisis, la didáctica es considerada como conducta ubicable en un conjunto de ámbitos de desempeño que tienen su origen en las características de la práctica por enseñar. Aunque cada práctica da origen a su didáctica, es posible describir de manera genérica los diferentes ámbitos de enseñanza en términos de habilidades y competencias para enseñar. Para conseguirlo es necesario definir los propósitos generales de la enseñanza, en términos conductuales. Posteriormente, se requiere describir qué se hace y en qué situaciones se hace algo para conseguir dichos propósitos. A continuación se presenta una propuesta conductual para el análisis de la conducta vinculada a la enseñanza que denominada Modelo Interconductual de Desempeño Didáctico (MIDD).

\section{Modelo Interconductual de Desempeño Didáctico}

La práctica didáctica tiene como propósitos fundamentales: 1) Mediar la interacción del alumno con los referentes disciplinarios, 2) Facilitar el ajuste del comportamiento del alumno a los criterios paradigmáticos, y 3) Auspiciar el desarrollo de habilidades y competencias disciplinarias en el alumno (Silva \& Morales, 2008; Carpio, Pacheco, Flores \& Canales, 1998; Carpio, Chávez, Silva, Cárdenas, Escobedo, León, Sánchez, \& Reyes, 2005). En este trabajo se describen siete ámbitos de desempeño con los cuales se cumplen estos propósitos y que conforman lo que denominaremos Modelo Interconductual de Desempeño Didáctico (MIDD):

\section{a) Planeación didáctica}

Es el ámbito en el que se desarrollan habilidades y competencias para describir tareas, actividades y situaciones en las que tendrá lugar la interacción con el aprendiz. Para ello, el que enseña debe responder a cuestionamientos sobre qué debe aprender alguien, para qué debe aprenderlo, bajo qué situaciones debe aprenderlo y cómo evidenciar que se ha aprendido. Es con este tipo de conducta con la que se elaboran, entre otras cosas, los objetivos generales y específicos de aprendizaje en un plan de estudios. En las instituciones educativas formales ser hábil o competente para elaborar estos objetivos es fundamental para el que enseña, porque con dichos objetivos se estructuran los planes de estudio que organizan la actividad educativa cotidiana. 


\section{b) Exploración competencial}

En este ámbito el que enseña debe identificar y evaluar el comportamiento de quien aprende, en términos de las habilidades y competencias precurrentes a la interacción didáctica. El propósito de esta exploración es hacer ajustes al plan elaborado. Nótese la relación entre las habilidades de exploración con las correspondientes a la planeación. De no ser hábil para la planeación didáctica quien enseña carecería de los criterios para determinar qué explorar del comportamiento de quienes aprenden, menos podría hacer ajustes ya que no habría plan didáctico respecto del cual hacerlos (e.g., Daza \& Moreno, 2010).

\section{c) Explicitación de criterios}

En este caso es necesario poner en contacto al aprendiz con los criterios disciplinarios y didácticos que debe satisfacer con su desempeño. Por criterios disciplinarios deben entenderse todos aquellos requerimientos conductuales que son exclusivos del ámbito de aquello que se está enseñando (e.g., lo que se debe decir y lo que no en la práctica). Por criterios didácticos deben entenderse aquellos requerimientos conductuales vinculados a la organización de las actividades didácticas cotidianas (e.g., a qué hora llegar, cómo relacionarse con quien enseña, cómo relacionarse con otros compañeros).

\section{d) Ilustración}

Es el ámbito en el que se describe al aprendiz las características de las habilidades de un experto en la disciplina para la solución de un problema específico. Es decir, quien enseña debe poner en contacto al que aprende con los elementos del comportamiento de otro, señalando en esta descripción: a) lo que hacía, b) la situación en la que lo hacía, y c) para qué lo hacía. Ilustrar consiste en relacionar lingüísticamente al que aprende con las habilidades de otro, incluso cuando ese otro es quien enseña (i.e. quien enseña se pone como ejemplo). Es conveniente en este punto advertir que ilustrar no consiste en la presentación vacía de ejemplos, que adquiere su verdadero valor didáctico cuando un ejemplar es analizado por quien aprende en términos de los elementos que conforman una habilidad. También es posible que el ejemplar bajo análisis sea representativo de formas inapropiadas de proceder y que sirvan didácticamente para evitar estas formas de comportamiento.

\section{e) Práctica supervisada}

En este ámbito se debe involucrar a quien aprende en situaciones controladas de solución de problemas. El control de estas situaciones permite supervisar y corregir 
momento a momento el desempeño de quien aprende. Las habilidades y competencias a desarrollar se vinculan con la regulación de aquellos que aprenden en el transcurso de la situación didáctica, reduciendo las posibilidades de un desempeño inefectivo, y aprovechando al máximo la situación en beneficio de su desarrollo conductual.

\section{f) Retroalimentación}

En este ámbito el aprendiz debe hacer contacto con su desempeño en un momento previo, quien enseña debe ser hábil y competente para conseguir dicho contacto. Quien enseña describe al aprendiz lo que hizo, para qué lo hizo y en qué situación lo hizo. También pueden describirse las variantes del hacer (el desempeño) del aprendiz, que en ese momento eran posibles y que sin embargo no se concretaron. En este sentido, retroalimentar es algo más complejo que decir "correcto" o "incorrecto", sino que implica habilitar al aprendiz para entrar en contacto con su propio comportamiento y sus variantes posibles. Los criterios con base en los cuales se supervisa y retroalimenta el desempeño provienen del plan didáctico original, sin el cual no se podría supervisar ni retroalimentar la conducta del aprendiz. Existe la posibilidad de que dicho plan, y por lo tanto los objetivos de aprendizaje, hayan sido realizados por alguien más. Es indispensable que exista ese plan didáctico y que la ilustración, la retroalimentación y la supervisión se apeguen a los criterios de logro establecidos en la situación (e.g., Gómez \& Insausti, 2004; Palacino, 2007).

\section{g) Evaluación}

En este ámbito es necesario contrastar el desempeño real de quien aprende con un desempeño esperado, propuesto como objetivo de aprendizaje en el plan inicial. En este contraste es necesario contar con un sistema de valoración que permita determinar el grado de similitud entre el desempeño esperado y el real. Por ejemplo, en instituciones educativas formales se proponen métricas para determinar numéricamente este grado de correspondencia. Vale decir que la finalidad didáctica de este contraste no solo descansa en la posibilidad de extender una calificación de la conducta, sino sobre todo en crear la posibilidad de realizar ajustes estratégicos a interacciones didácticas posteriores. La reprobación (entendida como la falta de correspondencia entre el desempeño real y el esperado), se constituye como un fracaso didáctico y debería poner en alerta a quien enseña. La reprobación significa que se ha fracasado al planificar, explorar, explicitar, ilustrar, supervisar y retroalimentar el comportamiento de quien aprende. En otras palabras, significa que no se ha mediado el contacto (i.e., no hay condiciones para la interacción), no se ha auspiciado el desarrollo, ni se ha facilitado el cumplimiento de los criterios para el aprendiz, esto 
es, que la interacción no ha sido "didáctica". A continuación se presenta la Tabla 1, en la que se describen de manera esquemática los ámbitos antes mencionados, ubicando su ejercicio en espacios educativos en instituciones formales.

Tabla 1

Ámbitos de desempeño didáctico en espacios educativos formales

\begin{tabular}{|c|c|c|c|}
\hline $\begin{array}{l}\text { Ámbitos de } \\
\text { desempeño }\end{array}$ & $\begin{array}{l}\text { Criterio } \\
\text { (¿Qué?) }\end{array}$ & $\begin{array}{l}\text { Situación } \\
\text { (¿Dónde?) }\end{array}$ & $\begin{array}{l}\text { Desempeño } \\
\text { (¿Cómo?) }\end{array}$ \\
\hline $\begin{array}{l}\text { Planeación } \\
\text { didáctica }\end{array}$ & $\begin{array}{l}\text { Elaborar planes. } \\
\text { Definir los objetivos } \\
\text { de aprendizaje. }\end{array}$ & Estudio, cubículo. & Escribe, proyecta, etc. \\
\hline $\begin{array}{l}\text { Exploración } \\
\text { competencial }\end{array}$ & $\begin{array}{l}\text { Identificar repertorio } \\
\text { de entrada en } \\
\text { estudiantes. }\end{array}$ & $\begin{array}{l}\text { Aula, taller, } \\
\text { laboratorio. }\end{array}$ & $\begin{array}{l}\text { Pregunta, planta } \\
\text { problemas, pide auto } \\
\text { reportes, etc. }\end{array}$ \\
\hline Criterios & $\begin{array}{l}\text { Media el contacto } \\
\text { del estudiante con } \\
\text { los criterios. }\end{array}$ & $\begin{array}{l}\text { Aula, taller, } \\
\text { laboratorio. }\end{array}$ & $\begin{array}{l}\text { Describe, ilustra, } \\
\text { entrega escritos, etc. }\end{array}$ \\
\hline Ilustración & $\begin{array}{l}\text { Media el contacto } \\
\text { del estudiante con } \\
\text { la solución de } \\
\text { problemas. }\end{array}$ & $\begin{array}{l}\text { Aula, taller, } \\
\text { laboratorio. }\end{array}$ & $\begin{array}{l}\text { Resuelve problemas } \\
\text { propios de la disciplina } \\
\text { frente al estudiante. }\end{array}$ \\
\hline $\begin{array}{l}\text { Supervisión de la } \\
\text { practica }\end{array}$ & $\begin{array}{l}\text { Regula la interacción } \\
\text { estudiante-problema. }\end{array}$ & $\begin{array}{l}\text { Aula, taller, } \\
\text { laboratorio. }\end{array}$ & $\begin{array}{l}\text { Variable según } \\
\text { el problema. }\end{array}$ \\
\hline Retroalimentación & $\begin{array}{l}\text { Auspicia la } \\
\text { interacción del } \\
\text { estudiante. }\end{array}$ & $\begin{array}{l}\text { Aula, taller, } \\
\text { laboratorio. }\end{array}$ & $\begin{array}{l}\text { Variable según las } \\
\text { características del } \\
\text { desempeño que } \\
\text { retroalimenta. }\end{array}$ \\
\hline Evaluación & $\begin{array}{l}\text { Determina la } \\
\text { correspondencia } \\
\text { del desempeño del } \\
\text { estudiante con los } \\
\text { criterios y objetivos } \\
\text { del programa. }\end{array}$ & $\begin{array}{l}\text { Donde tiene lugar } \\
\text { el desempeño del } \\
\text { estudiante. }\end{array}$ & $\begin{array}{l}\text { Contrastando } \\
\text { desempeño real con } \\
\text { el ideal esperado o } \\
\text { prescrito. }\end{array}$ \\
\hline
\end{tabular}


Como es previsible, las habilidades y competencias que se desarrollan en cada uno de estos ámbitos se expresan de diferentes maneras que dependen de los siguientes factores:

a) El grado de formalidad del espacio educativo. Estas interacciones deben estar cubiertas por objetivos de un sistema normativo social que le da pertinencia a lo que se enseña, por ejemplo no es lo mismo el ejercicio didáctico en el club de ajedrez que en las instituciones educativas más rígidas como las militares o las medicas Las formas más conocidas de los espacios educativos son aquellas vinculadas a las instituciones escolarizadas como los talleres, seminarios o laboratorios. A partir de sus características distintivas, cada espacio permite cierta configuración de una interacción didáctica.

b) El nivel educativo. En cada nivel existen propósitos diferenciados, por ejemplo, la educación básica tiene como propósito habilitar a las personas para la sobrevivencia y convivencia. Por su parte, la educación media superior tiene el propósito de habilitar a los individuos para la producción no especializada. Finalmente, la educación superior tiene como propósito habilitar a las individuos para la producción especializada y la transformación de las formas de producir (De Alba, 1997).

c) La disciplina en cuestión. Puede ser científica, tecnológica, técnica. Dichas dimensiones cuentan con sus propias reglas para realizarse (i.e. criterios conductuales por satisfacer), por lo que enfatiza diferencialmente el desarrollo de habilidades didácticas del que enseña.

d) La biografía conductual de quien enseña. En ella se pueden identificar estilos interactivos, habilidades y competencias desarrolladas anteriormente, preferencias y actitudes.

e) Los recursos disponibles para la interacción didáctica. Abarcan desde parámetros espaciales y temporales, así como los materiales, aparatos, instrumentos, las sesiones didácticas y su duración, y que incluyen también aspectos relacionados con la dinámica socio educativa en la que se ubica la labor didáctica.

Todos estos factores influyen en el desarrollo de las habilidades y competencias planteadas por el MIDD. Con base en este modelo se han realizado algunos estudios experimentales con el propósito de generar evidencia de su utilidad para comprender la labor didáctica. A continuación se describen brevemente algunos de ellos.

\section{Descripción de algunos estudios que respaldan el modelo}

Una vez que se propusieron los ámbitos del desempeño didáctico, el primer paso de investigación que se dio fue el de identificar habilidades y competencias en situaciones reales de enseñanza (Escobedo, 2006; Sáenz, 2006).Para ello se convocó a varios profesores universitarios a quienes se les pidió su autorización para video gra- 
bar sus clases. Se diseñó un sistema de evaluación con base en categorías de observación del desempeño docente, registrando lo que los profesores hicieron en cada uno de estos ámbitos. En general, los resultados de este primer estudio indicaron lo siguiente:

a) Se observaron diferencias en términos de las habilidades requeridas en cada profesor en el transcurso de las clases y entre profesores, en los ámbitos de la explicitación de criterios, ilustración y retroalimentación.

b) Las habilidades registradas con mayor frecuencia fueron aquellas vinculadas al ámbito de los criterios. En contraste, las habilidades registradas con menor frecuencia se ubicaron en el ámbito de la ilustración y retroalimentación.

En un trabajo posterior, Chávez (2005) diseñó una tarea experimental para evaluar habilidades en el ámbito de la planeación didáctica. En la tarea diseñada los profesores participantes debían identificar los componentes que conforman un plan de trabajo académico, elaborar dicho plan y expresar por escrito las características genéricas que definen a cualesquier plan. De manera general, los datos obtenidos indicaron que los profesores podían planificar sin que fueran capaces de decir cuáles son los criterios de la "planificación general" (características genéricas y formales que debería tener). Para la autora de este trabajo, los resultados cuestionaron la idea tradicional de que sólo se necesita el dominio conceptual de "los principios de la didáctica" para el hacer efectivo. Finalmente, Silva (2009) realizó un estudio para evaluar el grado y tipo de transferencia entre habilidades de enseñanza y habilidades científicas. Las habilidades de enseñanza que se consideró fueron las relativas a la creación de objetivos de aprendizaje para programas de cursos ficticios, mientras que las habilidades científicas que se consideró fueron aquellas vinculadas a la creación de objetivos de investigación. Silva se propuso evaluar el efecto de entrenar con base en un tipo de objetivo y probar el desempeño creando objetivos en otro ámbito. Adicionalmente, evaluó el efecto de tres tipos de entrenamiento: identificar los objetivos apropiados, elaborarlos y formular las características genéricas de los objetivos. De este modo, Silva entrenó a los participantes a resolver las tres tareas y evaluó la transferencia sólo con un tipo de tarea con objetivos de distinto ámbito. Los resultados de esta investigación indicaron que fue más sencillo para los participantes identificar objetivos que elaborarlos, un resultado en concordancia con el estudio previamente mencionado. También se encontró que fue más fácil durante el entrenamiento identificar y elaborar objetivos de investigación que objetivos de aprendizaje. Sin embargo, cuando los participantes debieron identificar o elaborar objetivos diferentes a los entrenados, el desempeño fue mejor para quienes fueron entrenados con base en objetivos de aprendizaje. El autor considera que estos resultados se deben en mayor medida a dos factores: 1 ) los reportes de investigación difirieron cualitativamente entre sí, en mayor medida que los programas didácticos, 2) Los programas didácticos 
hacían referencias a prácticas de investigación, y en el caso de los resúmenes de investigación no había relación con la organización didáctica de algún curso. En otras palabras, el entrenamiento pudo estar ligeramente sesgado a favor del desarrollo de habilidades para identificar, elaborar o formular objetivos de investigación. Sin embargo, los resultados del estudio realizado fueron sistemáticos, parecen indicar que en efecto son posibles relaciones de transferencia en el desarrollo de habilidades correspondientes a ámbitos o prácticas distintas.

En la actualidad se siguen desarrollando investigaciones con base en el MIDD que poco a poco irán generando mayor conocimiento sobre la forma en que los diferentes factores que constituyen una interacción didáctica modulan el desarrollo de habilidades para la enseñanza. Conforme se van acumulando evidencias sobre las características de las habilidades y competencias didácticas surgirán cuestionamientos a la teoría que les da sentido y surgirán también nuevos retos metodológicos por superar. A continuación se mencionan algunas de estas implicaciones.

\section{Conclusión}

Una de las ventajas de proponer el MIDD teniendo como base una teoría del desarrollo psicológico es que permite anticipar la existencia, al menos teórica, de tipos de conducta en estos ámbitos de desempeño. Por ejemplo, interconductualmente se propone que el desarrollo de competencias es la condición necesaria, aunque no suficiente, para el desarrollo de la creatividad. Existen espacios educativos formales que se constituyen como situaciones contingencialmente cerradas o abiertas que rara vez permiten el desarrollo de la conducta creativa. Esto suele ocurrir sobre todo en los niveles básicos de la enseñanza, en los que se espera que los aprendices desarrollen habilidades y competencias muy precisas. Por otra parte, en los espacios educativos que se ubican en los niveles superiores la enseñanza contempla en más ocasiones la posibilidad de promover en el aprendiz el comportamiento creativo. En estos casos las situaciones que enfrentan los aprendices son "contingencialmente abiertas". Es decir, hay múltiples problemas por resolver y cada uno de ellos soluble en múltiples formas. Esto podría propiciar que quien enseña en este nivel debe dominar una gama más amplia de situaciones y estrategias para la enseñanza, siendo proclive a proponer sus propias estrategias. Quizá para incrementar las probabilidades de éxito de conseguir que otra persona se comporte creativamente sea necesario ser competente y creativo. Casos representativos de lo anterior son la enseñanza y el aprendizaje de las disciplinas científicas, artísticas y tecnológicas. Sin embargo, prácticamente cualquier disciplina que requiere incorporar a individuos en la vanguardia de su conocimiento requiere por lo tanto de propiciar el comportamiento creativo en los individuos. De este modo, quienes enseñan y quienes aprenden contribuyen unos a otros, aunque 
de manera diferente, a su desarrollo psicológico. Así, el análisis de la conducta, amparada en esta propuesta, debería ahondar en estrategias para la promoción de comportamiento efectivo, variado e innovador en aquellos que se incorporan a la práctica, tanto científica, como tecnológica o técnica.

En la medida que se fortalezca este modelo, en el que enseñar se describe en términos de habilidades, competencias y creatividad en el desarrollo psicológico, se definirán estrategias metodológicas más contundentes y eficaces para probar el efecto de variables ya conocidas en la investigación básica. Entre los retos por superar se encuentran aquellos vinculados a la evidencia del comportamiento más complejo y que suele estar en relación con aspectos lingüísticos. Por ejemplo, el estudio de lo que piensa quien enseña en la interacción didáctica, o quizá, el mismo pensamiento como interacción didáctica. Otro de los retos por superar se relaciona con la controversia entre la validez ecológica de los estudios que se realizan bajo condiciones controladas de observación y las dificultades para realizar estas investigaciones en situaciones reales de enseñanza. Sin embargo, como ya se ha mencionado, el primer y el más importante de los pasos es contar con un modelo que permita progresar sistemáticamente.

En un futuro quizá, se puedan proponer estrategias aplicables para promover en quienes enseñan habilidades, competencias y creatividad didácticas, cada una de estas estrategias ad hoc a las necesidades de cada espacio y nivel. En la actualidad es preocupante que las actividades de actualización docente convoquen a muy pocas personas con la responsabilidad de enseñar y que el tipo de actividades para actualizarse sean mayoritariamente de tipo informativo. Este manuscrito es una modesta contribución, pero autentica en su interés de plantear en los lectores la inquietud de desarrollar investigación y aplicaciones a favor de la conducta didáctica.

\section{Referencias}

Carpio, C., Canales, C., Morales. H, Arroyo. R., \& Silva, H. (2007). Inteligencia, creatividad y desarrollo psicológico. Acta Colombiana de Psicología, 10(2). 41-50.

Carpio, C., Chávez Favela, M.C., Silva, H., Cárdenas, K., Escobedo, L., León, A., Sánchez, J., \& Reyes, R. (2005). Fundamentos teórico-metodológicos de un modelo interconductual de evaluación de habilidades y competencias docentes en educación de habilidades y competencias docentes en educación en educación superior. Memorias del XVII Congreso Mexicano de Análisis de la Conducta, San Luis Potosí, México.

Carpio, C., Pacheco, V., Canales, C., \& Flores, C. (1998). Comportamiento inteligente y juegos de lenguaje en la enseñanza de la psicología. Acta Comportamentalia, $6(1), 47-60$. 
Carpio, C., Pacheco, V., Canales, C., \& Flores, C. (2005). Aprendizaje de la Psicología: un análisis funcional. En C. Carpio, \& J. Irigoyen (Eds.), Psicología y Educación: Aportaciones desde la teoría de la conducta. (pp.1-32). México: UNAM.

Chávez, M. (2005). Evaluación de habilidades y competencias docentes en el ámbito de la planeación didáctica un estudio con docentes universitarios. (Tesis de Maestría, Universidad de Sonora. División de Ciencias Sociales, Sonora, México). Recuperada de http://www.bibliotecas.uson.mx/sib_acervo.aspx

Daza-Pérez, E.P. \& Moreno-Cárdenas, J.A. (2010). El pensamiento del profesor de ciencias en ejercicio: Concepciones sobre la enseñanza y el aprendizaje de las ciencias naturales. Revista Electrónica de Enseñanza de las Ciencias, 9(3), 549568. Recuperado dehttp://www.saum.uvigo.es/reec/volumenes/volumen9/ART4_ Vol9_N3.pdf

De Alba, A. (1997). El currículum universitario ante los retos del siglo XXI: La paradoja entre posmodernidad, ausencia de utopía y determinación curricular. En: A. De Alba (Ed), El currículum universitario: De cara al nuevo milenio (pp. 29-45). México: Plaza y Valdés.

Escobedo, L. (2006). Diseño de un sistema de evaluación de las habilidades docentes en las instituciones de educación superior. (Tesis licenciatura, Universidad Nacional Autónoma de México. Facultad de Estudios Superiores Iztacala, México). Recuperada de http://132.248.9.9:8080/tesdig/Procesados_2006/0602825/Index. html

Engels, F. (1988). El papel del trabajo en la transformación del mono en hombre. México, D.F.: Quinto Sol.

Gómez, J. A., \& Insausti, M. J. (2004). El ciclo reflexivo cooperativo: un modelo didáctico para la enseñanza de las ciencias. Revista Electrónica de Enseñanza de las Ciencias, 3(2), 148-160. Recuperado de http://www.saum.uvigo.es/reec/volumenes/volumen3/Numero2/ART2_Vol3_N2.pdf

López, F. (1994). Cultura y convenciones: un análisis Interconductual. En: L. Hayes, E. Ribes y V. López (Eds.) Psicología Interconductual, contribuciones en honor a J.R. Kantor (pp. 127-142). México: Universidad de Guadalajara.

Palacino, F. (2007). Competencias comunicativas, aprendizaje y enseñanza de las Ciencias Naturales: un enfoque lúdico. Revista Electrónica de Enseñanza de las Ciencias, 6(2): 275-298. Recuperado de http://www.saum.uvigo.es/reec/volumenes/volumen6/ART4_Vol6_N2.pdf

Sáenz, A. (2006). Análisis funcional de las estrategias didácticas. (Tesis de licenciatura, Universidad Nacional Autónoma de México. Facultad de Estudios Superiores Iztacala, México). Recuperada de http://132.248.9.9:8080/tesdig/Procesados_2006/0607441/Index.html

Silva, H. (2009). El desarrollo de habilidades de investigación científica y planeación 
didáctica: redefiniendo el vínculo entre la investigación y la docencia. (Tesis de Maestría. Universidad Nacional Autónoma de México. Facultad de Filosofía y Letras, México). Recuperada de http://132.248.9.195/ptd2009/mayo/0643411/Index. html

Silva, H.,\& Morales, G. (2008). Ejercicio y didáctica, la relevancia de los grupos de investigación. En C. Carpio (Ed.) Competencias profesionales del psicólogo: Investigación, experiencias y propuestas (pp.59-80). México: FES Iztacala UNAM.

Ribes, E. (1990). Problemas conceptuales en el Análisis del Comportamiento Humano. México: Trillas.

Ryle, G. (2005). El concepto de lo mental. México: Paidós Ibérica. 